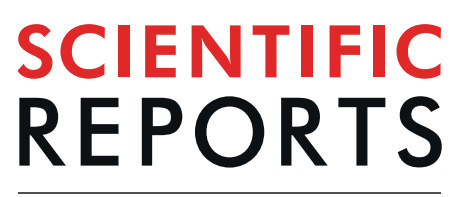

\title{
Effect of High-dose Antithrombin Supplementation in Patients with Septic Shock and Disseminated Intravascular Coagulation
}

\author{
Youn-Jung Kim ${ }^{1}$, Byuk Sung Ko ${ }^{2}$, Seo Young Park ${ }^{3}$, Dong Kyu Oh${ }^{4}$, Sang-Bum Hong ${ }^{4}$, \\ Seongsoo Jang ${ }^{5} \&$ Won Young Kim ${ }^{1 *}$
}

The efficacy of antithrombin (AT) administration in patients with septic shock and disseminated intravascular coagulation (DIC) was uncertain. This study aimed to investigate whether high-dose AT administration improves outcomes in patients with septic shock and DIC. This observational, prospective cohort study included consecutive adult septic shock patients with DIC who showed AT activity $<70 \%$ between March 2016 and August 2018. The 28 day mortality of the patients treated with AT and without AT was evaluated by propensity score matching and inverse probability of treatment weighting. Among 142 patients with septic shock and DIC, 45 patients (31.7\%) received AT supplementation and 97 did not. The 28 day mortality rate was lower in the AT group, but no statistically significant difference persisted after matching. Multivariable analysis showed that AT supplementation was independently associated with 28 day mortality (odds ratio [OR], $0.342 ; 95 \%$ $\mathrm{Cl}$ [confidence interval], $0.133-0.876 ; \mathrm{P}=0.025)$; however, no such association was observed after matching (OR, $0.480 ; 95 \% \mathrm{Cl}, 0.177-1.301 ; \mathrm{P}=0.149$ ). High-dose AT administration in septic shock patients with DIC showed the improvement in survival, but the improvement was not observed after matching. Further larger studies are needed to conclusively confirm these findings.

Sepsis is a heterogenous syndrome, defined as life-threatening organ dysfunction caused by a dysregulated host response to infection, and sepsis and septic shock represent a major global health problem ${ }^{1,2}$. Despite recent improvements in the understanding of the pathophysiology of sepsis and advances in clinical management, patient outcomes remain poor and have not significantly improved ${ }^{3}$. Currently, early, appropriate administration of antibiotics, fluid, and vasopressors are the only pharmacologic treatments shown to be associated with a survival benefit in these patients ${ }^{1}$. Additional adjuvant therapies are therefore required to optimize treatment and to individualize patient management according to their accompanying organ dysfunction.

Disseminated intravascular coagulation (DIC) is a frequent complication of septic shock and is associated with a high level of mortality ${ }^{1,4,5}$. In patients with septic shock, an impaired physiologic anticoagulant mechanism, particularly decreased antithrombin (AT) activity, leads to excessive microthrombus formation, microcirculatory dysfunction and, therefore, organ dysfunction ${ }^{6}$. AT is an important physiologic anticoagulant that affects the intrinsic, extrinsic, and common coagulation pathways, as well as exerting anti-inflammatory effects ${ }^{7,8}$. Despite the theoretical benefits of AT supplementation, current clinical practice guidelines do not support the use of AT in patients with severe sepsis and septic shock. These recommendations are based on data from the Phase III KyberSept clinical trial, which demonstrated that AT supplementation had no beneficial effect on overall mortality and that treatment was associated with an increased risk of bleeding ${ }^{1,8}$. However, a post hoc analysis and

${ }^{1}$ Department of Emergency Medicine, Asan Medical Center, University of Ulsan College of Medicine, 88 Olympic-ro 43-gil, Songpa-gu, Seoul, 05505, Korea. ${ }^{2}$ Department of Emergency Medicine, College of Medicine, Hanyang University, 222 Wangsimni-ro, Seongdong-gu, Seoul, 133-791, Korea. ${ }^{3}$ Department of Clinical Epidemiology and Biostatistics, Asan Medical Center, University of Ulsan College of Medicine, 88 Olympic-ro 43-gil, Songpa-gu, Seoul, 05505, Korea. ${ }^{4}$ Department of Pulmonary and Critical Medicine, Asan Medical Center, University of Ulsan College of Medicine, 88 Olympic-ro 43-gil, Songpa-gu, Seoul, 05505, Korea. ${ }^{5}$ Department of Laboratory Medicine, Asan Medical Center, University of Ulsan College of Medicine, 88 Olympic-ro 43-gil, Songpa-gu, Seoul, 05505, Korea.

*email:wonpia73@naver.com 


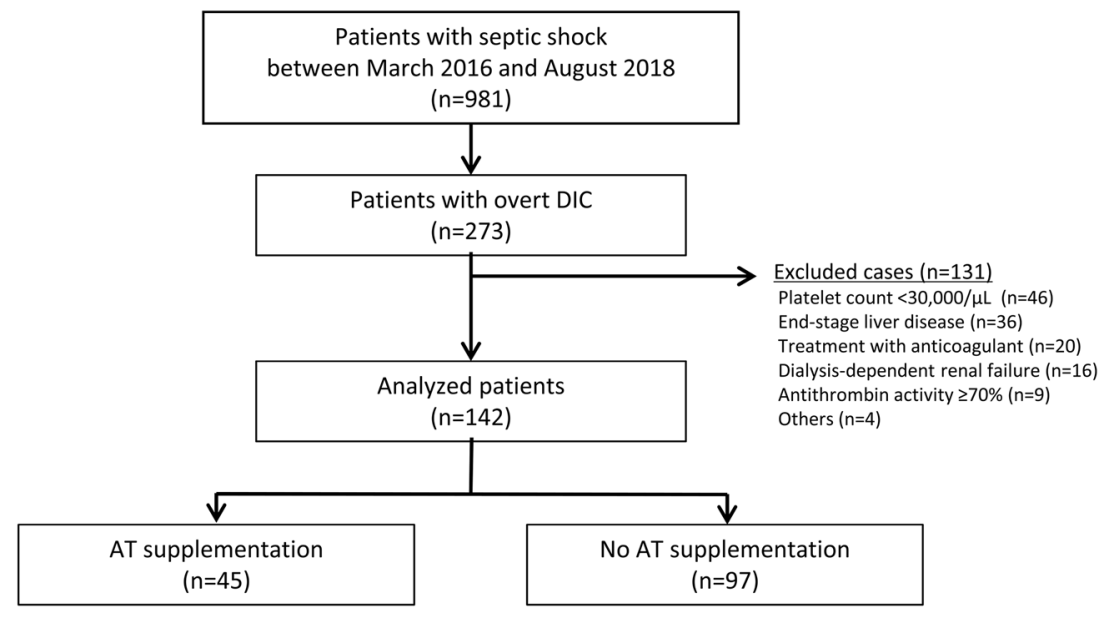

Figure 1. Patient flow diagram. Abbreviations: DIC, disseminated intravascular coagulation.

systematic reviews have shown the possible benefit of AT in specific subgroups of patients, such as those with severe sepsis with DIC who did not receive concomitant heparin ${ }^{9-11}$. Uncertainty surrounding the efficacy of AT supplementation in these patients has resulted in inconsistency in clinical practice ${ }^{12}$. Therefore, this prospective cohort study aimed to investigate whether high-dose AT administration improves the clinical outcomes of patients with septic shock associated with DIC during the early phase of the condition.

\section{Results}

From a total of 981 patients with septic shock treated between March 2016 and August 2018, 273 were diagnosed with overt DIC (International Society on Thrombosis and Hemostasis [ISTH] score $\geq 5$ ) and were enrolled in this study; 131 patients who were not indicated for AT supplementation were excluded. Therefore, a total of 142 patients were included in the analysis; 45 patients received AT supplementation and 97 patients did not (Fig. 1). Propensity score matching was performed in 68 patients $(\mathrm{N}=34$ in each group).

The baseline and clinical characteristics did not differ significantly between the AT supplementation and no AT supplementation groups (Table 1). The median patient age was 67.5 years (interquartile ranges [IQR], $59.0-76.0)$, and 87 patients $(61.3 \%)$ were male. The most common infection focus was that of the hepatobiliary system (46.5\%), followed by respiratory infection (16.9\%). The median maximum sequential organ failure assessment (SOFA) and acute physiology and chronic health evaluation (APACHE) II scores were 10.0 (IQR, 7.8-12.0) and 20.0 (IQR, 14.8-24.3), respectively. Two thirds (66.2\%) of the study patients showed overt DIC at ED presentation. The median DIC score was higher in the AT supplementation group than the no AT supplementation group, although this difference did not reach statistical significance (median, 6.0 vs. 5.0, $\mathrm{P}=0.051$ ). The baseline and clinical characteristics of the study patients after propensity matching and inverse probability of treatment weighting (IPTW) are presented in Supplementary Tables 2 and 3, respectively.

Table 2 compares the clinical outcomes before and after propensity score matching. Prior to matching, the 28 day mortality was significantly lower in the AT supplementation group than the no AT supplementation group $(17.8 \%$ vs. $34.0 \%, P=0.047)$, while other secondary clinical outcomes showed no statistically significant differences. In the propensity-matched cohort, all clinical outcomes (including 28 and 90 day mortality, recovery of organ function, and changes in SOFA score) showed no statistically significant differences between the two groups. No major bleeding events were seen in either group for 7 days.

The odds ratios (ORs) of AT supplementation for clinical outcomes including 28 day mortality, 90 day mortality, and recovery of organ function in patients with septic shock and DIC are presented in Fig. 2. The logistic regression analysis of crude, propensity score-matched, and IPTW propensity data demonstrated that AT supplementation was not significantly associated with any of the clinical outcomes. In the overall cohort, AT supplementation significantly improved 28 day mortality (OR, $0.34 ; 95 \% \mathrm{CI}, 0.13-0.88 ; \mathrm{P}=0.03$ ). This tendency towards reduced 28 day mortality in patients with AT supplementation was still observed in propensity score-matched groups $(\mathrm{OR}, 0.69 ; 95 \% \mathrm{CI}, 0.21-2.22 ; \mathrm{P}=0.531)$ and IPTW propensity data $(\mathrm{OR}, 0.42 ; 95 \% \mathrm{CI}$, $0.18-1.30 ; \mathrm{P}=0.149)$, respectively, although without reaching statistical significance.

\section{Discussion}

In this prospective observational study, propensity score matching among the patients with septic shock and DIC who showed AT activity $<70 \%$ showed no significant difference in 28 day mortality between patients receiving AT supplementation and those who did not. Despite the theoretical benefits of AT supplementation for patients with septic shock, this approach did not result in an improvement in clinical outcomes with respect to mortality and the recovery of organ function after propensity score analyses (matching and IPTW). However, patients who received AT supplementation had no serious bleeding events. Despite the low statistical power, this study provides information that can serve as a basis for clinical equipoise to support the need of further investigation and possibly randomized clinical trial. 


\begin{tabular}{|c|c|c|c|c|}
\hline Characteristics & $\begin{array}{l}\text { Total } \\
\mathrm{n}=142\end{array}$ & $\begin{array}{l}\text { AT supplementation } \\
\mathrm{n}=45\end{array}$ & $\begin{array}{l}\text { No AT supplementation } \\
\mathrm{n}=97\end{array}$ & P-value \\
\hline \multicolumn{5}{|l|}{ Comorbid disease } \\
\hline Age, years & $67.5(59.0-76.0)$ & $67.0(54.0-76.0)$ & $68.0(60.0-76.5)$ & 0.588 \\
\hline Male & $87(61.3)$ & $22(48.9)$ & $65(67.0)$ & 0.039 \\
\hline Hypertension & $50(35.2)$ & $16(35.6)$ & $34(35.1)$ & 0.953 \\
\hline Diabetes mellitus & $31(21.8)$ & $8(17.8)$ & $23(23.7)$ & 0.426 \\
\hline Metastatic solid cancer & $72(50.7)$ & $22(48.9)$ & $50(51.5)$ & 0.768 \\
\hline Other comorbid disease $^{\mathrm{a}}$ & $7(4.9)$ & $0(0)$ & $7(7.2)$ & 0.097 \\
\hline Infection focus & & & & 0.105 \\
\hline Respiratory system & $24(16.9)$ & $4(8.9)$ & $20(20.6)$ & \\
\hline Hepatobiliary system & $66(46.5)$ & $26(57.8)$ & $40(41.2)$ & \\
\hline Others & $52(36.6)$ & $15(33.3)$ & $37(38.1)$ & \\
\hline \multicolumn{5}{|l|}{ Laboratory findings } \\
\hline White blood cell count, $/ \mu \mathrm{L}$ & $8050(4150-17825)$ & $8300(4850-15850)$ & $7700(3500-18250)$ & 0.585 \\
\hline Hemoglobin, g/dL & $10.6(2.15)$ & $10.6(2.36)$ & $10.6(2.05)$ & 0.966 \\
\hline Platelet, $\times 10^{3} / \mu \mathrm{L}$ & $81.0(47.8-129.8)$ & $78.0(46.0-142.0)$ & $85.0(51.0-128.5)$ & 0.488 \\
\hline Prothrombin time, INR & $1.53(1.35-1.74)$ & $1.56(1.42-1.72)$ & $1.51(1.34-1.78)$ & 0.593 \\
\hline Sodium, $\mathrm{mmol} / \mathrm{L}$ & $133.9(5.87)$ & $133.9(5.03)$ & $134.0(6.25)$ & 0.923 \\
\hline Potassium, mmol/L & $4.2(3.6-4.7)$ & $4.1(3.5-4.7)$ & $4.2(3.6-4.8)$ & 0.266 \\
\hline Chloride, $\mathrm{mmol} / \mathrm{L}$ & $98.5(6.62)$ & $98.6(6.52)$ & $98.5(6.71)$ & 0.950 \\
\hline Creatinine, mg/dL & $1.49(1.07-2.29)$ & $1.20(1.02-2.29)$ & $1.55(1.17-2.31)$ & 0.066 \\
\hline Albumin, g/dL & $2.4(2.0-2.7)$ & $2.3(2.1-2.8)$ & $2.4(2.0-2.7)$ & 0.918 \\
\hline $\mathrm{CRP}, \mathrm{mg} / \mathrm{dL}$ & $15.00(6.48-21.96)$ & $14.48(5.22-21.75)$ & $15.89(6.59-22.78)$ & 0.535 \\
\hline Lactic acid, mmol/L & $4.7(2.9-7.4)$ & $4.2(2.5-6.4)$ & $4.9(3.2-7.7)$ & 0.112 \\
\hline \multicolumn{5}{|l|}{ Severity score } \\
\hline SOFA score & $10.0(7.8-12.0)$ & $9.0(7.0-12.0)$ & $10.0(8.0-12.0)$ & 0.201 \\
\hline APACHE II score & $20.0(14.8-24.3)$ & $20.0(13.5-25.5)$ & $20.0(16.0-24.0)$ & 0.787 \\
\hline DIC documentation & & & & 0.399 \\
\hline At presentation & $94(66.2)$ & $32(71.1)$ & $62(63.9)$ & \\
\hline$<24$ hours after admission & $48(33.8)$ & $13(28.9)$ & $35(36.1)$ & \\
\hline DIC score by ISTH criteria & $5.0(5.0-6.0)$ & $6.0(5.0-6.0)$ & $5.0(5.0-6.0)$ & 0.051 \\
\hline Antithrombin level, \% & $45.5(34.0-55.0)$ & $44.0(35.0-53.5)$ & $46.0(33.0-55.0)$ & 0.550 \\
\hline
\end{tabular}

Table 1. Baseline and clinical characteristics of the study patients. Data are shown as median (interquartile range) or as $\mathrm{n}(\%) .{ }^{\mathrm{a}}$ Other comorbid disease includes coronary artery disease, chronic pulmonary disease, liver cirrhosis, chronic kidney disease, and previous cerebrovascular accident. Abbreviations: APACHE, acute physiology and chronic health evaluation; AT, antithrombin; CRP, C-reactive protein; DIC, disseminated intravascular coagulation; INR, international normalized ratio; ISTH, International Society on Thrombosis and Hemostasis; SOFA, sequential organ failure assessment.

\begin{tabular}{|l|l|l|l|l|l|l|}
\hline & \multicolumn{2}{|l|}{ Overall cohort } & \multicolumn{3}{l|}{ Propensity-matched cohort } \\
\cline { 2 - 7 } Clinical Outcomes & $\begin{array}{l}\text { AT supplementation } \\
\mathbf{n = 4 5}\end{array}$ & $\begin{array}{l}\text { No AT supplementation } \\
\mathbf{n = 9 7}\end{array}$ & P-value & $\begin{array}{l}\text { AT supplementation } \\
\mathbf{n}=\mathbf{3 4}\end{array}$ & $\begin{array}{l}\text { No AT supplementation } \\
\mathbf{n}=\mathbf{3 4}\end{array}$ & P-value \\
\hline 28 day mortality & $8(17.8)$ & $33(34.0)$ & 0.047 & $8(23.5)$ & $9(26.5)$ & 0.786 \\
\hline 90 day mortality & $16(35.6)$ & $45(48.4)$ & 0.155 & $14(41.2)$ & $14(41.2)$ & 0.754 \\
\hline Recovery of organ function & $35(77.8)$ & $68(70.1)$ & 0.340 & $26(76.5)$ & $27(79.4)$ & 0.777 \\
\hline Changes in SOFA score & $4.0(2.0-7.0)$ & $4.0(0.0-7.0)$ & 0.342 & $4.0(2.0-7.0)$ & $5.0(2.0-7.8)$ & 0.317 \\
\hline
\end{tabular}

Table 2. Comparisons of Clinical Outcomes. Data are shown as median (interquartile range) or as n (\%). Abbreviations: AT, antithrombin; SOFA, sequential organ failure assessment.

Although DIC frequently accompanies sepsis and is associated with a high rate of mortality, the main therapeutic strategy remains to treat the underlying causative factor without other adjunct treatment ${ }^{5}$. AT supplementation has been suggested as a potential adjunct for the treatment of sepsis-associated DIC on the basis of post hoc analysis of the KyberSept trial and retrospective observational studies ${ }^{8,10,13,14}$. In addition, a recent multicenter retrospective observational study by Hayakawa et al. has suggested that AT supplementation for patients with sepsis-induced DIC may reduce the rate of in-hospital mortality ${ }^{12}$. However, these data were not statistically robust, with only IPTW propensity score analysis showing a significant association between AT supplementation and low in-hospital mortality $(\mathrm{OR}, 0.75 ; 95 \% \mathrm{CI}, 0.57-0.98 ; \mathrm{P}=0.03)$; by contrast, a non-significant association 


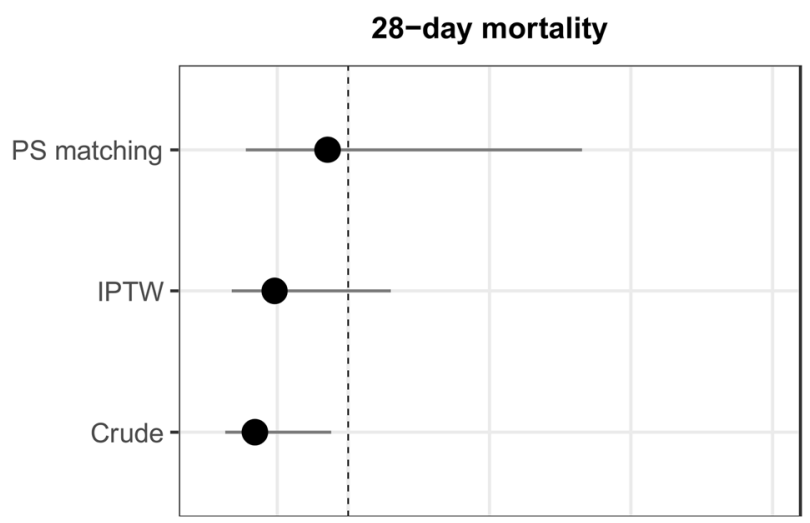

90-day mortality

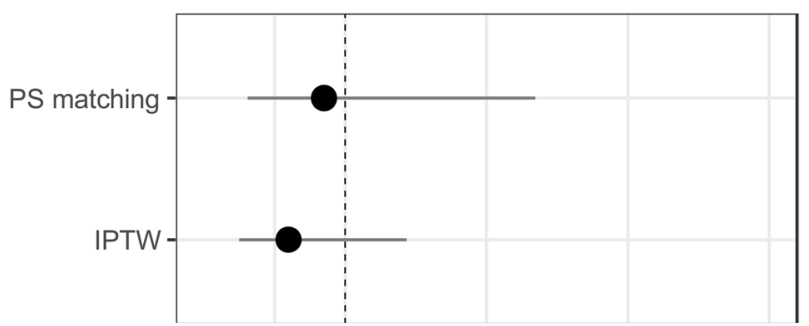

Recovery of organ dysfunction

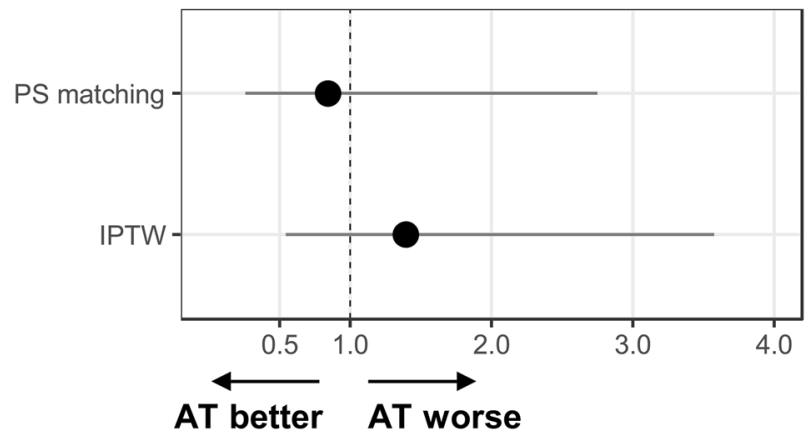

OR $(95 \% \mathrm{Cl})$

$0.86(0.28-2.65)$

$0.48(0.18-1.30)$

$0.34(0.13-0.88)$

$0.85(0.31-2.35)$

$0.60(0.25-1.43)$

$0.84(0.26-2.75)$

$1.40(0.54-3.58)$

Figure 2. Odds ratios for clinical outcomes for antithrombin supplementation. Abbreviations: AT, antithrombin; CI, confidence interval; IPTW, inverse probability of treatment-weighted; PS, propensity score.

was seen using quintile-stratified propensity score analysis and propensity score matching analysis ${ }^{12}$. Due to these conflicting results, the beneficial effect of AT supplementation on survival in patients with sepsis and DIC remains controversial.

In the current study, we aimed to evaluate the beneficial effect of AT supplementation in patients with septic shock and overt DIC who showed AT activity $<70 \%$. Based on the results of previous studies, this target group was considered to be most likely to benefit from adjunct treatment ${ }^{8,9,11,12,14}$. In a crude analysis, patients treated with or without AT supplementation showed no statistically significant differences in baseline and clinical characteristics. With respect to mortality, the crude 28 day mortality of the patient cohort was $28.9 \%$ (41/142), which is lower than that reported in previous studies of patients with severe sepsis and DIC, and those with septic shock and DIC, which have shown a 28 day mortality rate of $30-70 \%^{4,14}$. In the current study, there was an absolute difference of 14.2 percentage points in the crude model in favor of AT supplementation versus no AT supplementation, with the AT supplementation group showing a higher survival rate than those without (34.0 vs $17.8 \%$, $\mathrm{P}=0.047$ ). When a randomized controlled trial is not possible, propensity score matching and IPTW analysis is a powerful approach to estimate treatment effect in observational studies, reducing treatment selection bias and the inherent limitations of an observational study ${ }^{15}$. After matching was carried out in the current study, AT supplementation in patients with septic shock and DIC showed a consistent tendency towards reducing mortality but the statistical significance of AT supplementation was diminished. This may have been due to the reduced number of study patients in the matched group. Decreased AT activity is another important prognostic factor in patients with sepsis and DIC, and AT supplementation has been seen to have a greater beneficial effect in patients with decreased AT activity, particularly in those with values $<40 \%{ }^{14,16}$. Hayakawa et al. recently suggested that patients with sepsis and DIC who had very low AT activity $(<43 \%)$ were ideal candidates for AT supplementation, rather than those with AT activity $<70 \%{ }^{17}$. In the current study, the median AT activity of our study patients 
was $45.5 \%$, and this relatively high level of AT activity may have contributed to the diminished beneficial effect of AT supplementation.

Our study has several limitations. First, it was conducted in a single center, which limits the generalizability of the results. Secondly, the small sample size could mean that the statistical analyses are underpowered. The relatively lower 28 day mortality rate and the limited sample size would contribute the lack of power, and might have contributed to the lack in significance of the results. Larger studies are needed to conclusively confirm these findings. The study design did not include the recovery of DIC or elevation of AT activity as secondary outcomes, but instead included a patient-centered outcome, 28 day mortality, as the primary outcome. Although recovery of DIC or improvement of AT activity would be important clinical outcomes, mortality is more clinically relevant. The study collected data only on major bleeding events, which were defined as intracranial or those requiring a transfusion of at least three units of blood. Although this approach did not collect data on minor bleeding events and consequently reduces the understanding of adverse events, it was considered to be a less subjective approach that could reduce diagnostic errors and ascertainment bias. In addition, although the attending physicians treated the patients according to the established guidelines, interventions such as the administration of appropriate antibiotics and low-dose hydrocortisone, the timing of administration of antibiotics, continuous renal replacement therapy and mechanical ventilation or other blinded variables could affect the clinical outcomes and be potential confounding factors.

In conclusion, AT supplementation for patients with septic shock and overt DIC who showed AT activity $<70 \%$ was associated with improved survival with no increased risk of major bleeding event. However, this association was not seen after propensity matching and IPTW. Considering the heterogeneity of septic shock, larger studies are needed to conclusively confirm these findings and to identify candidates most likely to benefit from AT supplementation.

\section{Materials and Methods}

Study design and patient selection. This observational, prospective, cohort study of adult patients with septic shock and DIC was conducted in the emergency department (ED) of a university-affiliated, tertiary hospital in Seoul, Korea between March 2016 and August 2018. The septic shock registry has prospectively collected data for all consecutive adult patients (aged $\geq 19$ years) with septic shock at EDs since June $2012^{18}$. Patients with septic shock were defined as those with refractory hypotension (systolic blood pressure $<90 \mathrm{mmHg}$, mean arterial pressure $<70 \mathrm{mmHg}$, or a systolic blood pressure decrease $>40 \mathrm{mmHg}$ ) requiring vasopressor treatment despite receiving at least $20-30 \mathrm{~mL} / \mathrm{kg}$ intravenous crystalloid, or those with hypoperfusion (blood lactate concentration $\geq 4 \mathrm{mmol} / \mathrm{L}$ ) with suspected or confirmed infection ${ }^{19,20}$. The septic shock registry did not include patients assigned as 'do not attempt resuscitation', those transferred from other hospitals after treatment for septic shock, patients transferred directly from the ED to other hospitals, or those developing septic shock 6 hours after ED presentation.

Data from patients admitted with a diagnosis of septic shock and DIC were included in the analysis. Additional blood coagulation tests, including fibrinogen and fibrinogen degradation products, were routinely performed after November $2014^{4}$, and the measurement of AT activity level has been performed in patients with septic shock between 9 a.m. and 5 p.m. on weekdays since March 2016. DIC was defined in accordance with the criteria outlined by the ISTH (Supplementary Table 1), i.e., an ISTH DIC score $\geq 5^{21}$. Patients with an ISTH DIC score $\geq 5$ and documented AT activity $<70 \%$ were treated with AT (30 IU $/ \mathrm{kg}$ for the first day and $3000 \mathrm{IU} /$ day for the next two consecutive days) within 6 hours of diagnosis. Patients who met any of the following criteria were not treated with AT and were excluded from the study: AT activity $\geq 70 \%$; evidence of bleeding; treatment with heparin (except subcutaneous low dose or intravenous line flushing) or oral anticoagulants; a platelet count $<30,000 / \mu \mathrm{L}$; immunocompromised status; acute myocardial infarction (within the previous 7 days); preexisting dialysis-dependent renal failure; end-stage liver disease; transplantation (postoperative state); history of stroke within the last year; history of hypersensitivity to AT; third-degree burns ( $\geq 20 \%$ of the total body area); and pregnancy ${ }^{8}$. Patients were categorized into AT supplementation or no AT supplementation groups. Patients in the no AT supplementation group comprised those individuals who could not undergo laboratory testing for AT activity within 6 hours of septic shock and DIC recognition as this facility was unavailable.

The study design was approved by the institutional review board of Asan Medical Center and informed consent was obtained prior to data collection (study no: 2016-0205). All procedures performed in this study were in accordance with the ethical standards of the institutional research committee and with the 1964 Helsinki Declaration and its later amendments or comparable ethical standards.

Management and data collection. All patients with septic shock received treatment in accordance with the current 6 hour bundles of survival sepsis campaign, including the administration of intravenous crystalloid, obtaining blood cultures prior to the administration of antibiotics and the administration of broad-spectrum antibiotics and vasopressors ${ }^{19}$. Transfusion, including fresh frozen plasma or platelets, was not performed in the absence of significant bleeding, significant risk of bleeding, or planned invasive procedures ${ }^{19}$. Unfractionated or low molecular weight heparin was used to treat venous thrombosis and heparin was used for intravenous line flushing. The decision to include further interventions, such as mechanical ventilation and continuous renal replacement therapy, was based on standard institutional intensive care protocols under the direction of the attending intensive care physician.

The demographic and clinical characteristics of the patients (including age, sex, comorbid disease, focus of infection, laboratory findings, and severity scores such as SOFA and APACHE II scores) and clinical outcomes (such as 28 day mortality and in-hospital mortality) were retrieved from the septic shock registry. SOFA and APACHE II scores were calculated using the worst parameters within 24 hours of ED admission ${ }^{22,23}$. Recovery of organ function was defined as a decrease in SOFA score $\geq 2$ from the first day to 7 days after ED presentation. 
ISTH scores and AT level were also collected. Major bleeding events, defined as intracranial or those requiring transfusion of $\geq 3$ units of blood, were recorded for 7 days 8 .

The primary outcome of the study was 28 day mortality. Secondary outcomes were 90 day mortality, recovery of organ function within 7 days, and changes in SOFA score for 7 days after admission or until in-hospital death.

Statistical analyses. Descriptive statistics (percentages; medians and IQR) were used to present participant characteristics overall, with chi-square tests or the Mann-Whitney U-test used to assess differences in patient characteristics between the AT supplementation and no AT supplementation groups. Because there were systematic differences between the two groups, two methods were considered to remove the confounding effect from the AT supplementation effect: propensity score matching and IPTW. These methods provide unbiased estimates of the treatment effect by creating a pseudo-population in which the treatment is like-randomized ${ }^{24-26}$. First, the propensity score (probability of receiving AT supplementation) was estimated for each patient by logistic regression using all covariates shown in Table 1. Patients who had similar propensity scores were matched in a 1:1 ratio, with caliper of 0.2 times the standard deviation of the logit of the propensity score, resulting in 34 propensity score-matched pairs. For IPTW, we assigned each patient a stabilized weight, which was the product of the marginal probability of receiving AT supplementation and the inverse of the propensity score for patients in the AT supplementation group. For patients in the no AT supplementation group, the stabilized weight was the product of the marginal probability of not receiving AT supplementation and the inverse of (1-propensity score). Propensity score-matched data and the data with IPTW were checked for group comparability using the standardized mean difference.

The effect of AT supplementation was evaluated using three methods: 1. crude multivariable logistic analysis, 2. analysis of propensity score-matched data, and 3. analysis of data with IPTW. In the crude analysis, multivariable logistic regression was conducted on the original data and was interpreted as the association between AT supplementation and outcomes. In the analyses of propensity score-matched or weighted data, logistic or linear regression was performed and the resulting OR estimates were interpreted as a closer approximation to the causal effect of the AT supplementation. All statistical analyses were performed using R 3.5.1 (R Foundation for Statistical Computing, Vienna, Austria).

\section{Data availability}

The datasets used and/or analysed during the current study are available from the corresponding author on reasonable request.

Received: 14 June 2019; Accepted: 23 October 2019;

Published online: 12 November 2019

\section{References}

1. Rhodes, A. et al. Surviving sepsis campaign: international guidelines for management of sepsis and septic shock: 2016 . Intensive care medicine 43, 304-377 (2017).

2. Singer, M. et al. The third international consensus definitions for sepsis and septic shock (Sepsis-3). Jama 315, 801-810 (2016).

3. Rhee, C. et al. Incidence and trends of sepsis in US hospitals using clinical vs claims data, 2009-2014. Jama 318, 1241-1249 (2017).

4. Ko, B. S. et al. The Prevalence and Significance of Overt Disseminated Intravascular Coagulation in Patients with Septic Shock in the Emergency Department According to the Third International Consensus Definition. The Korean. Journal of Critical Care Medicine 31, 334-341 (2016).

5. Papageorgiou, C. et al. Disseminated Intravascular Coagulation: An Update on Pathogenesis, Diagnosis, and Therapeutic Strategies. Clinical and applied thrombosis/hemostasis: official journal of the International Academy of Clinical and Applied Thrombosis/ Hemostasis, 1076029618806424 (2018).

6. De Backer, D., Orbegozo Cortes, D., Donadello, K. \& Vincent, J.-L. Pathophysiology of microcirculatory dysfunction and the pathogenesis of septic shock. Virulence 5, 73-79 (2014).

7. Harada, N., Okajima, K., Kushimoto, S., Isobe, H. \& Tanaka, K. Antithrombin reduces ischemia/reperfusion injury of rat liver by increasing the hepatic level of prostacyclin. Blood 93, 157-164 (1999).

8. Warren, B. L. et al. High-dose antithrombin III in severe sepsis: a randomized controlled trial. Jama 286, 1869-1878 (2001).

9. Umemura, Y., Yamakawa, K., Ogura, H., Yuhara, H. \& Fujimi, S. Efficacy and safety of anticoagulant therapy in three specific populations with sepsis: a meta-analysis of randomized controlled trials. Journal of thrombosis and haemostasis: JTH 14, 518-530 (2016).

10. Kienast, J. et al. Treatment effects of high-dose antithrombin without concomitant heparin in patients with severe sepsis with or without disseminated intravascular coagulation. Journal of thrombosis and haemostasis: JTH 4, 90-97 (2006).

11. Wiedermann, C. J. \& Kaneider, N. C. A systematic review of antithrombin concentrate use in patients with disseminated intravascular coagulation of severe sepsis. Blood coagulation \& fibrinolysis: an international journal in haemostasis and thrombosis 17, 521-526 (2006).

12. Hayakawa, M. et al. Antithrombin supplementation and mortality in sepsis-induced disseminated intravascular coagulation: a multicenter retrospective observational study. Shock 46, 623-631 (2016).

13. Tagami, T., Matsui, H., Fushimi, K. \& Yasunaga, H. Supplemental dose of antithrombin use in disseminated intravascular coagulation patients after abdominal sepsis. Thrombosis and haemostasis 114, 537-545 (2015).

14. Iba, T., Saito, D., Wada, H. \& Asakura, H. Efficacy and bleeding risk of antithrombin supplementation in septic disseminated intravascular coagulation: a prospective multicenter survey. Thrombosis research 130, e129-133 (2012).

15. Streiner, D. L. \& Norman, G. R. The pros and cons of propensity scores. Chest 142, 1380-1382 (2012).

16. Iba, T., Saitoh, D., Wada, H. \& Asakura, H. Efficacy and bleeding risk of antithrombin supplementation in septic disseminated intravascular coagulation: a secondary survey. Critical care (London, England) 18, 497 (2014).

17. Hayakawa, M., Yamakawa, K., Kudo, D. \& Ono, K. Optimal Antithrombin Activity Threshold for Initiating Antithrombin Supplementation in Patients With Sepsis-Induced Disseminated Intravascular Coagulation: A Multicenter Retrospective Observational Study. Clinical and applied thrombosis/hemostasis: official journal of the International Academy of Clinical and Applied Thrombosis/Hemostasis 24, 874-883 (2018).

18. Kim, Y.-J. et al. Platelet-Lymphocyte Ratio After Granulocyte Colony Stimulating Factor Administration: An Early Prognostic Marker in Septic Shock Patients with Chemotherapy-Induced Febrile Neutropenia. Shock (2018). 
19. Dellinger, R. P. et al. Surviving Sepsis Campaign: international guidelines for management of severe sepsis and septic shock, 2012. Intensive care medicine $39,165-228$ (2013).

20. Casserly, B. et al. Lactate measurements in sepsis-induced tissue hypoperfusion: results from the Surviving Sepsis Campaign database. Critical care medicine 43, 567-573 (2015).

21. Levi, M., Toh, C., Thachil, J. \& Watson, H. Guidelines for the diagnosis and management of disseminated intravascular coagulation. British journal of haematology 145, 24-33 (2009).

22. Vincent, J.-L. et al. The SOFA (Sepsis-related Organ Failure Assessment) score to describe organ dysfunction/failure. Intensive care medicine 22, 707-710 (1996).

23. Knaus, W. A., Draper, E. A., Wagner, D. P. \& Zimmerman, J. E. APACHE II: a severity of disease classification system. Critical care medicine 13, 818-829 (1985).

24. Austin, P. C. \& Stuart, E. A. Moving towards best practice when using inverse probability of treatment weighting (IPTW) using the propensity score to estimate causal treatment effects in observational studies. Statistics in medicine 34, 3661-3679 (2015).

25. Austin, P. C. An introduction to propensity score methods for reducing the effects of confounding in observational studies. Multivariate behavioral research 46, 399-424 (2011).

26. Rosenbaum, P. R. \& Rubin, D. B. The central role of the propensity score in observational studies for causal effects. Biometrika 70, $41-55(1983)$.

\section{Author contributions}

W.Y.K. designed the study; Y.J.K. and B.S.K. collected data; Y.J.K. and S.Y.P. analyzed data; Y.J.K., D.K.O., S.B.H., S.J. and W.Y.K. interpreted data; Y.J.K., S.Y.P. and W.Y.K. wrote the paper; and all authors reviewed and approved the final manuscript.

\section{Competing interests}

Won Young Kim has received honoraria from Green Cross. The remaining authors declare that they have no competing interests. Green Cross did not engage the design of the study and collection, analysis, interpretation of data and writing the manuscript.

\section{Additional information}

Supplementary information is available for this paper at https://doi.org/10.1038/s41598-019-52968-y.

Correspondence and requests for materials should be addressed to W.Y.K.

Reprints and permissions information is available at www.nature.com/reprints.

Publisher's note Springer Nature remains neutral with regard to jurisdictional claims in published maps and institutional affiliations.

(c) (1) Open Access This article is licensed under a Creative Commons Attribution 4.0 International License, which permits use, sharing, adaptation, distribution and reproduction in any medium or format, as long as you give appropriate credit to the original author(s) and the source, provide a link to the Creative Commons license, and indicate if changes were made. The images or other third party material in this article are included in the article's Creative Commons license, unless indicated otherwise in a credit line to the material. If material is not included in the article's Creative Commons license and your intended use is not permitted by statutory regulation or exceeds the permitted use, you will need to obtain permission directly from the copyright holder. To view a copy of this license, visit http://creativecommons.org/licenses/by/4.0/.

(c) The Author(s) 2019 